\title{
COMPORTAMIENTO ASINTÓTICO DE LA ECUACIÓN DE ONDA CON CONDICIÓN DE FRONTERA DEL TIPO NEWMANN, LOCALMENTE DISTRIBUIDO
}

\author{
Alfonso Pérez Salvatierra ${ }^{1}$ \& Victoriano Yauri Luque ${ }^{2}$ \\ William César Olano Díaz ${ }^{3}$ \& José Quique Broncano ${ }^{4}$ \\ Víctor Hilario Tarazona Miranda ${ }^{5}$
}

\begin{abstract}
Resumen.- Se estudia el comportamiento asintótico de la ecuación de onda con condición de frontera del tipo Newmann, localmente distribuido. Empleando el Principio de la Continuación Única, resultado estudiado por Ruiz [5] y aplicado a problemas de comportamiento asintótico por Zuazua [6].
\end{abstract}

Palabras claves: Decaimiento exponencial, multiplicadores, continuación única.

\section{ASYMPTOTIC BEHAVIOR OF THE WAVE EQUATION WITH BOUNDARY CONDITION OF NEWMANN TYPE, LOCALLY DISTRIBUTED}

\begin{abstract}
In this note we shall study the asymptotic behavior of the wave equation with boundary condition of Newmann type, locally distributed. Using the Principle of the Unique Continuation, result studied by Ruiz [5] and applied to problems of asymptotic behavior by Zuazua [6].
\end{abstract}

Key words: Exponential decay, multipliers, unique continuation.

\footnotetext{
${ }^{1}$ UNMSM, Facultad de Ciencias Matemáticas, Lima - Perú, e-mail:aperezs@unmsm.edu.pe ${ }^{2}$ UNMSM, Facultad de Ciencias Matemáticas, Lima - Perú, e-mail:vyauril@unmsm.edu.pe ${ }^{3}$ UNMSM, Facultad de Ciencias Matemáticas, Lima - Perú, e-mail:wcolano@hotmail.com ${ }^{4}$ UNMSM, Facultad de Ciencias Matemáticas, Lima - Perú, e-mail: jquiqueb@unmsm.edu.pe ${ }^{5}$ UNMSM, Facultad de Ciencias Matemáticas, Lima - Perú, e-mail:vtarazonam@unmsm.edu.pe
} 


\section{INTRODUCCIÓN}

En esta sección plantearemos las hipótesis y algunos resultados necesarios para el desarrollo del presente trabajo.

(1) H 1. $\Omega \subset \mathbb{R}^{n}$ es un abierto, acotado $(n \geq 1), \Gamma=\partial \Omega$ frontera de clase $C^{2}$.

(2) H 2. $a(x) \in L_{+}^{\infty}(\Omega), a(x) \geq a_{0}>0$ c.s. en $\omega$, donde $\omega \subseteq \Omega$ es una vecindad de $\Gamma=\partial \Omega$.

(3) H 3. $f$ es tal que $f(s) s \geq 0, \forall s \in \mathbb{R}$ y además $f$ es super lineal, es decir,

$$
\exists \delta>0 ; f(s) s \geq(2+\delta) F(s) ; \forall \in \mathbb{R}
$$

(4) H 4. $f$ tiene la propiedad del crecimiento, es decir,

$$
|f(x)-f(y)| \leq C\left[1+|x|^{p-1}+|y|^{p-1}\right]|x-y| ; \forall x, y \in \mathbb{R}
$$

y algún $(C<0, p>1)$ con $(n-2) p \leq n$.

(5) H 5. $F(s)=\int_{0}^{s} f(z) d z \geq 0$, pues $f(s) s \geq 0 ; \forall s \in \mathbb{R}$.

(6) H 6. Para un $x_{0} \in \mathbb{R}^{n}$ fijo se tiene que $\partial \Omega=\Gamma=\bar{\Gamma}_{0} \cup \bar{\Gamma}_{1}$ donde

$$
\Gamma_{0}=\{x \in \Gamma ; m(x) \cdot v(x)>0\}, \Gamma_{1}=\{x \in \Gamma ; m(x) \cdot v(x)<0\}
$$

con $m(x)=x-x_{0}, v(x)$, normal unitario en un punto $x$.

Con las hipótesis H 1 - H 6, se estudiará el sistema siguiente

$$
(*) \mid \begin{array}{ll}
u_{t t}-\Delta u+u+f(u)+a(x) u_{t}=0 & \text { en } \Omega \times(0,+\infty) \\
\frac{\partial u}{\partial \nu}=0 & \text { sobre } \Gamma \times(0,+\infty) \\
u(0)=u^{0} \in H^{1}, u_{t}(0)=u^{1} \in L^{2}(\Omega) & \text { en } \Omega
\end{array}
$$

donde $a(x) \geq a_{0}>0$ c.s. en $\omega, \omega \subseteq \Omega$ vecindad de $\Gamma=\partial \Omega$.

Se tiene que el problema $(*)$, está bien puesto en el espacio $H_{0}^{1}(\Omega) \times L^{2}(\Omega)$, es decir, para datos iniciales $\left\{u^{0}, u^{1}\right\} \in H_{0}^{1}(\Omega) \times L^{2}(\Omega)$, existe solución única débil de $(*)$ en la 
clase $u \in C\left([0,+\infty) ; H_{0}^{1}(\Omega)\right) \cap C^{-1}\left([0,+\infty) ; L^{2}(\Omega)\right)$. Para una prueba de ello se puede utilizar el método de Faedo-Galerkin o la Teoría de Semigrupo de Operadores.

Podemos ver, referencias con aplicaciones a la Física en los resultados de Komornik [2], Muñoz [7], Pérez [8], Cabanillas [10], Portillo [11].

Se define la energía del sistema $(*)$ como:

$$
E(t)=\frac{1}{2} \int_{\Omega}\left\{\left|u_{t}(x, t)\right|^{2}+|\nabla u(x, t)|^{2}+|u(x, t)|^{2}\right\} d x+\int_{\Omega} F(u(x, t)) d x
$$

El objetivo del trabajo es obtener el decaimiento uniforme exponencial de la energía para soluciones del problema $(*)$, es decir,

$$
\exists C, \gamma>0 \text { tal que } E(t) \leq C e^{-\gamma t}, \forall t \in \cdot(0,+\infty)
$$

\section{RESULTADOS PREVIOS}

Basado en el siguiente resultado de A. Ruiz [5], se tiene el siguiente lema, Lema (resultado de la continuación única): Sean $b \in L^{\infty}(\omega \times(0, T))$, $w \in H^{1}(\Omega \times(0, T))$ tal que $w$ satisface,

$$
(* *) \mid \begin{array}{ll}
w^{n}-\Delta w+b(x, t) w=0 & \text { en } \Omega \times(0, t)=Q \\
w=0 & \text { sobre } \Sigma=\Gamma \times(0, T) \\
w=0 & \text { casi siempre en } \omega \times(0, T)
\end{array}
$$

Entonces $w \equiv 0$.

Este resultado caracteriza el método de la continuación única, que consiste en que $w$ es solución débil del sistema (**), el cual es cero en una vecindad de la frontera, esto es suficiente para que $w$ sea cero en todo el cuerpo $\Omega \subset \mathbb{R}^{n}$.

Deducción de la energía formal:

Multiplicando al sistema $(*)$ por $u_{t}$ se obtiene:

$$
\frac{d}{d t}\left\{\frac{1}{2}\left|u_{t}\right|^{2}+\frac{1}{2}|\nabla u|^{2}+\frac{1}{2}|u|^{2}+\int_{\Omega} F(u) d x\right\}=-\int_{\Omega} a(x)\left|u_{t}\right|^{2} d x
$$


de la hipótesis (2) se tiene que $\int_{\Omega} a(x)\left|u_{t}\right|^{2} d x>0$, entonces

$$
\frac{d}{d x} E(t)<0, \forall t \in(0,+\infty)
$$

esto es, la energía dado en (7) es decreciente en $(0,+\infty)$.

Usando la técnica de los multiplicadores se obtiene la estimativa de la energía siguiente:

Para $T>0$ suficientemente grande;

(9)

$$
E(t) \leq C\left\{\int_{0}^{T} \int_{\Omega} a(x)\left|u_{t}(x, t)\right|^{2} d x d t+\int_{0}^{T} \int_{\Omega}|u(x, t)|^{2} d x d t\right\}
$$

Usando técnica de la continuación única pasmado en el lema precedente se obtiene la estimativa.

$$
\int_{0}^{T} \int_{\Omega}|u(x, t)|^{2} d x d t \leq C \int_{0}^{T} \int_{\Omega} a(x)|u(x, t)|^{2} d x d t
$$

Combinando (9) y (10) se obtiene que: $\exists C>0$ tal que:

$$
E(t) \leq C \int_{0}^{T} \int_{\Omega} a(x)\left|u_{t}(x, t)\right|^{2} d x d t
$$

De (11) y propiedad de semigrupo se obtiene el teorema central, el decaimiento exponencial, es decir,

$$
\exists C, \gamma>0 \text { tal que } E(t) \leq C E(0) e^{-\gamma t}
$$

\section{MÉTODOS Y RESULTADOS}

Los métodos y resultados seguidos son:

1. Técnica de los multiplicadores:

Sirvió para obtener la energía y otras estimativas para la energía.

2. Técnicas de las desigualdades integrales: 
Sirvió para obtener estimados de la integral de la energía como es,

$$
\int_{0}^{T} E(t) \leq C \int_{\Sigma_{0}} m \cdot v\left[\left|u_{t}\right|^{2}+F(u)\right]+|\widehat{x}|+\int_{\Omega}\left|(a(x) u) m \cdot \nabla u_{t}\right|
$$

donde $\Sigma_{0}=\Gamma_{0} \times(0, T), Q=\Omega \times(0, T) \mathrm{y}$

$$
\widehat{x}=\left(\int_{\Omega}\left(u_{t}+a(x) u\right) m \cdot \nabla u+\alpha u\left(u_{t}+\frac{a(x) u}{2}\right)\right]_{0}^{T}
$$

3. Técnica de la continuación única:

Nos sirvió para estimar el término (10)

4. Usando propiedades de semigrupo, finalmente obtenemos el decaimiento de la energía dado por (12).

\section{ANÁLISIS Y DISCUCIÓN}

Para $\Omega \subset \mathbb{R}^{n}$ abierto regular de clase $C^{2}$, consideremos la ecuación de la onda no homogénea:

$$
(\bar{*}) \mid \begin{array}{ll}
\theta^{\prime \prime}-\Delta \theta=f & \text { en } Q=\Omega \times[0, T] \\
\theta(0)=\theta^{0}, \theta^{t}(0)=\theta^{1} & \text { en } \Omega \\
\frac{\partial \theta}{\partial v}=0 & \text { sobre } \Sigma=\Gamma \times[0, T]
\end{array}
$$

Lema 1: Sea $\Omega$ un dominio acotado de $\mathbb{R}^{n}$ con frontera $\Gamma$ de clase $C^{2}, q=\left(q_{k}\right)_{k \geq 1}$ un campo vectorial de clase $\left[C^{1}(\Omega)\right]^{n}$.

Entonces, para toda solución débil $\theta=\theta(x, t)$ del sistema $(\bar{*})$ (es decir, $\left.\forall\left\{\theta^{0}, \theta^{1}\right\} \in H_{0}^{1}(\Omega) \times L^{2}(\Omega), f \in L^{1}\left(0, T ; L^{2}(\Omega)\right)\right)$

Se tiene:

$$
\begin{aligned}
\int_{\Omega}\left(\theta^{\prime \prime}-\Delta \theta\right) q \cdot \nabla \theta= & -\frac{1}{2} \int_{\Sigma} q \cdot v\left|\theta^{\prime}\right|^{2}+\left(\int_{\Omega} \theta^{\prime} q \cdot \nabla \theta\right]_{0}^{T}+ \\
& +\frac{1}{2} \int_{Q} \operatorname{div} \cdot(q)\left[\left|\theta^{t}\right|^{2}-|\nabla \theta|^{2}\right]+\int_{Q} \frac{\partial\left(q_{k}\right)}{\partial x_{j}} \frac{\partial \theta}{\partial x_{k}} \frac{\partial \theta}{\partial x_{j}}
\end{aligned}
$$


Demostración: Usando la técnica de los multiplicadores, multiplicando a $(\bar{*})$ por $q_{k} \frac{\partial \theta}{\partial x_{k}}$, donde $\theta$ es la solución débil del sistema $(\bar{*})$ se obtiene el lema.

Lema 2: Para u solución de (*) y con las hipótesis dados en (1) - (2) se tiene

$$
\begin{aligned}
\frac{1}{2} \int_{Q} \operatorname{div} \cdot(q) & {\left[\left|\theta_{t}\right|^{2}-|\nabla \theta|^{2}-2|u|^{2}-2 F(u)\right]+\int_{Q} q \cdot \nabla u\left[a(x) u_{t}-u\right]+} \\
& +\int_{Q} \frac{\partial u}{\partial x_{j}} \frac{\partial u}{\partial x_{k}} \frac{\partial}{\partial x_{j}}\left(q_{k}\right)=\frac{1}{2} \int_{\Sigma} q \cdot v\left[\left|\theta_{t}\right|^{2}-2|u|^{2}-2 F(u)\right]-\left(\int_{\Omega} u_{t} q \cdot \nabla u\right]_{0}^{T}
\end{aligned}
$$

Demostración: Usando la técnica de los multiplicadores, multiplicar a (*) por $q . \nabla u$.

Observación: El lema precedente es también válido para $q \in\left(W^{1, \infty}(\Omega)\right)^{n}$.

Lema 3: Con las hipótesis dadas en (1), (2) y (6) se tiene:

$$
\begin{aligned}
\left(\int_{\Omega} m \cdot \nabla u_{t}+a(x) u\right]_{0}^{T} & +\frac{n}{2} \int_{Q}\left|u_{t}\right|^{2}+\left(1-\frac{n}{2}\right) \int_{Q}|\nabla u|^{2}-n+\int_{Q}|u|^{2}+n \int_{Q} F(u)+ \\
& -\int_{Q} a(x) u m \cdot \nabla u_{t}-\int_{Q} u m \cdot \nabla u \leq \int_{\Sigma_{0}} m \cdot v\left[\left|u_{t}\right|^{2}-|u|^{2}+F(u)\right]
\end{aligned}
$$

Lema 4: Sea $\Omega \subset \mathbb{R}^{n}$ regular y u solución débil de $(*)$, se tiene:

$$
\left(\int_{\Omega} u\left(u_{t}+\frac{a(x) u}{2}\right)\right]_{0}^{T}=\int_{Q}\left[\left|u_{t}\right|^{2}-|\nabla u|^{2}\right]-\int_{Q}\left[u f(u)+|u|^{2}\right]
$$

Demostración: Multiplicando por $\xi u$ al sistema $(*)$ con $\xi \in W^{1, \infty}(\Omega)$ y luego, tomar $\xi=1$.

$\underline{\text { Lema 5: }}$ Con los hipótesis de (1) - (6), se tiene que, $\exists C>0$ tal que:

$$
C \int_{0}^{T} E(t) \leq \int_{\Sigma_{0}} m \cdot v\left|\left[\left|u_{t}\right|^{2}+|u|^{2}+F(x)\right]\right|+|\widehat{x}|+\int_{Q}\left|a(x) u m \cdot \nabla u_{t}\right|
$$

donde $\widehat{x}=\left(\int_{\Omega}\left\{m \cdot \nabla u\left[u_{t}+a(x) u\right]+\alpha u\left(u_{t}+\frac{a(x) u}{2}\right)\right\}\right]_{0}^{T}, \alpha>0$ 
Demostración: Combinando los lemas (3) y (4) se obtiene el lema precedente.

Lema 6: Con las hipótesis de (1) - (4) se obtiene

$$
\left(\int_{\Omega} u\left(u_{t}+\frac{a(x) u}{2}\right)\right]_{0}^{T}=\int_{Q}\left[\left|u_{t}\right|^{2}-|\nabla u|^{2}\right]-\int_{Q}|u|-\int_{Q} u f(u)
$$

Demostración: Multiplicación por $\xi(x)$ u $a(x)$ al sistema $(*)$ e integrado de 0 a $T$.

Proposición 1: Con la hipótesis de (1) - (6) se obtiene,

$$
T E(T) \leq C_{1}\left\{\int_{Q} a(x)\left|u_{t}\right|^{2}+\int_{Q}|u|^{2}+\left|\left(\int_{\Omega} u_{t} h \cdot \nabla u\right]_{0}^{T}\right|+|\widehat{x}|+|\widehat{y}|\right\}
$$

$\operatorname{con} \widehat{y}=\left(\int_{\Omega} \eta\left(u_{t}+\frac{a(x) u}{2}\right)\right]_{0}^{T}$

Demostración: Resulta de los lema 6, lema 5 y además desde que, la energía es decreciente, obteniendo;

$$
T E(T) \leq \int_{0}^{T} E(t) d t
$$

Lema 7: Con la hipótesis de (1) - (4) se tiene:

$$
|\widehat{x}|+|\widehat{y}|+\left|\left(\int_{\Omega} u_{t} h \cdot \nabla u\right]_{0}^{T}\right| \leq C_{2}\left(2 E(T)+\int_{Q} a(x)\left|u_{t}\right|^{2}\right)
$$

Proposición 2: Con las hipótesis de (1) - (6) obtenemos,

$$
E(T) \leq C\left(\int_{Q} a(x)\left|u_{t}\right|^{2}+\int_{Q}|u|^{2}\right), C>0
$$


Demostración: Del lema 7 en la proposición 1:

$$
\begin{aligned}
& T E(T)\left.\leq C_{1}\left\{\int_{Q} a(x)\left|u_{t}\right|^{2}+\int_{Q}|u|^{2}\right\}+C_{1}\left\{\mid \int_{\Omega} u_{1} h \cdot \nabla u\right]_{0}^{T}|+| \widehat{x}|+| \widehat{y} \mid\right\} \\
& \leq C_{1}\left\{\int_{Q} a(x)\left|u_{t}\right|^{2}+\int_{Q}|u|^{2}\right\}+C_{1} C_{2}\left(2 E(T)+\int_{Q} a(x)\left|u_{t}\right|^{2}\right) \\
& \leq C_{3}\left\{\int_{Q} a(x)\left|u_{t}\right|^{2}+\int_{Q}|u|^{2}+2 E(T)+\int_{Q} a(x)\left|u_{t}\right|^{2}\right\}, C_{3}=\max \left\{C_{1}, C_{1} C_{2}\right\} \\
&\left(T-2 C_{3}\right) E(T) \leq K\left\{\int_{Q} a(x)\left|u_{t}\right|^{2}+\int_{Q}|u|^{2}\right\}, K=\max \left\{2 C_{3}, C_{3}\right\} \\
& E(T) \leq \frac{K}{T-2 C_{3}}\left\{\int_{Q} a(x)\left|u_{t}\right|^{2}+|u|^{2}\right\}
\end{aligned}
$$

Para $T$ suficientemente grande tal que $T-2 C_{3}>0$, obtenemos la proposición 2 .

Proposición 3: Con las hipótesis de (1) - (4) obtenemos,

$$
\int_{Q}|u|^{2} \leq C \int_{Q} a(x)\left|u_{t}\right|^{2}
$$

Demostración: Su demostración es por el absurdo, usando el principio de la continuación única (ver A. Ruiz [5]), construyendo un sistema estacionario cuya solución lleva a una contradicción.

\section{TEOREMA CENTRAL}

Sea $\Omega \subset \mathbb{R}^{n}$ abierto, limitado con frontera de clase $C^{2}$, y se satisfacen las condiciones de (1) - (6). Entonces, existen constantes $C, \gamma>0$ tal que,

$$
E(t) \leq C E(0) e^{-\gamma t}, \forall t \geq 0
$$

y para toda solución débil de $(*)$. 
Demostración: De la proposición 3 en proposición 2 se tiene que, $\exists C_{0}>0$ tal que:

$$
E(T) \leq C_{0} \int_{0}^{T} \int_{\Omega} a(x)\left|u_{t}\right|^{2} d x d t
$$

desde que,

$$
\frac{d}{d t} E(t)=-\int_{\Omega} a(x)\left|u_{t}\right|^{2} d x
$$

integrando de 0 a $T$ :

$$
E(T)-E(0)=-\int_{0}^{T} \int_{\Omega} a(x)\left|u_{t}\right|^{2} d x d t
$$

Combinando (13) y (14) se obtiene:

$$
E(T) \leq \frac{C_{0}}{1+C_{0}} E(0), C=\frac{C_{0}}{1+C_{0}}<1, T>0
$$

Luego, por la propiedad se semigrupo y como el problema $(*)$ está bien puesto, obtenemos:

$$
E(t) \leq K E(0) e^{-\gamma t}, \forall t \geq 0
$$

para toda solución débil de $(*)$, donde

$$
C=\frac{C_{0}}{1+C_{0}}, \gamma=\frac{1}{T} \log C ; K=\frac{C_{0}}{1+C_{0}}
$$

\section{COMENTARIOS}

Existen muchos métodos para el estudio del decaimiento de una ecuación de la onda, con condiciones iniciales y de frontera. Nosotros abordamos el estudio del sistema $(*)$ gracias al gran resultado obtenido por Ruíz [5], el principio de la Continuación única usando el método indirecto (reducción al absurdo), técnicas de los multiplicadores y técnicas de las desigualdades integrales. 


\section{CONCLUCIONES}

El método usado para obtener el decaimiento exponencial del sistema (*), hemos visto que es, usando el principio de la continuación única, según A. Ruiz [5], puede también adaptarse a otros sistemas a los cuales se les puede agregar o variar datos de la frontera. Además es posible aplicar este método a otros modelos, comos son: Von Karman con condiciones de disipación en la frontera, placas con condiciones mixtas en la frontera de tipo Dirichlet - Newmann, etc., que podrían ser materias de estudios.

El método usado es unas de las múltiples maneras de poder obtener el decaimiento exponencial de la energía de ciertos sistemas dados.

\section{REFERENCIAS}

[1] A. Haraux, Stabilization of trayectories for some wealkly damped hyperbolic equations, J. Differential equation 59 (1985), 145 - 154.

[2] V. Komornik, Exact controllability in short time for the wave equation, Ann. Inst. H. Poincaré, Analyse Nonlinéaire 6 (1989), pag. 153 - 164.

[3] V. Komornik, and E. Zuazua, Stabilizatión frontiére de I'équation des ondes: Une Méthode directe, C. R. Acad. Sci. Paris 305 (1987) pag. 605 - 608.

[4] J. L. Lions, Contrâlabilité exacte, perturbations et stabilisations de systémes Distribués. Tome 1. Contrôlabilité Exacte", RMA 8, Masson, 1988.

[5] A. Ruiz, Unique continuation for weak solution of the wave equation plus a potencial Jornal Math pure Applicada 71, 1992 pag. 455 - 467.

[6] Zuazua, Enrike: Exponential decay for the semilinear wave equation with locally distribuited damping. Comm PDE, 15, pp 205 - 235 (1990).

[7] Muñoz R. J. Bisognin. V. - Bisognin. E. Exponential decay to partially thermoelastic, materiales. (1996).

[8] Pérez, Alfonso: Decaimento de solucoes de equacoes parcialmente Viscoelásticas. Tesis Doctorado UFRJ. Brasil (1997).

[9] Pérez, Alfonso: Decaimiento exponencial da la solución de onda con potencial y amortiguamiento localmente distribuido. Proy. Inv. Inst. de Invest. UNMSM. Revista Pesquimat. 2004. Lima - Perú.

[10] Cabanillas, Eugenio: Estabilización de la energía para una ecuación de Kirchoff Con disipación Localizada, Proy. Invest. Inst. Invest. UNMSM. 2004. Lima - Perú.

[11] Portillo, Higinio - Muñoz, Jaime: Sobre un problema de contacto unidimensional de Ondas elásticas localmente Amortecidas. SBA 46 Seminario Brasileiro de Análisis 1997. Brasil. 